\title{
Análise estocástica de uma empresa do setor siderúrgico
}

\author{
Stochastic analysis of a steel firm
}

\author{
Guilherme Freitas Cardoso 1 \\ Guilherme Santos Souza ${ }^{2}$ \\ Luciano Ferreira Carvalho ${ }^{3}$ \\ Karem Cristina de Sousa Ribeiro ${ }^{4}$
}

\begin{abstract}
Resumo
Avaliar empresas se apresenta como uma função gerencial capaz de verificar como a tomada de decisões atua na criação de valor e na competitividade das empresas. A utilização de métodos estocásticos permite ampliar a análise em relação aos métodos determinísticos, pois possibilita a mensuração da volatilidade do mercado por meio de fatores probabilísticos e aleatórios demonstrados em probabilidades de ocorrência. Sendo assim, este estudo tem como objetivo verificar o comportamento do valor presente do fluxo de caixa da Gerdau S.A. para os próximos cinco anos (2017-2021) por meio da utilização do método de Monte Carlo. A aplicação deste método possibilitou atribuir aspectos estatísticos para evidenciar os fatores correspondentes aos riscos do setor siderúrgico. Após os choques de simulação de cenários aleatórios, foi possível inferir que os métodos estocásticos permitem uma avaliação mais acurada. Como resultado demonstrou uma probabilidade de ocorrência de fluxo de caixa descontado negativo de $24 \%$ e de a Gerdau S.A. superar a expectativa determinística em $50,21 \%$, informações negligenciadas pelos métodos determinísticos.
\end{abstract}

Palavras-chave: Valor Presente do Fluxo de Caixa. Método Estocástico. Risco

\begin{abstract}
Firms valuation presents itself as a managerial function able to check out how the decisions making act over value creation and competitiveness of the firms. The use of stochastics methods allows to extend the analysis in relation to deterministic methods, because it makes possible measure the market volatility through probabilistic and random factors represented on probability of occurrence. Thus, this study aims to ascertain the behavior of the present value of cash flow of Gerdau S.A. to the next five years (2017-2021) using the Monte Carlo method. The application of this method allowed to attributestatistical factors to ascertain aspects of the risks of the steel sector. After the shocks of simulations of random scenarios, it was possible to infer that the stochastic methods allow a more accurate evaluation. It demonstrated a probability of negative discounted cash flow of $24 \%$ and that Gerdau S.A. surpasses the deterministic expectation in $50.21 \%$, information neglected by deterministic methods.
\end{abstract}

Keywords: Present Value of Cash Flow. Stochastic Method. Risk

\section{Introdução}

Ao avaliar empresas, os gestores se utilizam de ferramentas para tratamento dos dados visando vantagens competitivas que respaldem as tomadas de decisões, otimizando recursos e processos. Corroborando, Andreeva e Kianto (2012) relacionaram que maiores níveis de informação refletem de forma positiva no desempenho, bem como na competitividade, das empresas.

Mestrando em Finanças pela Universidade Federal de Uberlândia. Brasil. Afiliação: Universidade Federal de Uberlândia. Lattes: http://lattes. cnpq.br/7127739998677431 Email: gui-freitas@hotmail.com ORCID ID: http://orcid.org/0000-0002-9266-4220

2 Mestrando em Administração com ênfase em Finanças pela Universidade Federal de Uberlândia. Brasil. Afiliação: Universidade Federal de Uberlândia. Lattes: http://lattes.cnpq.br/8358528670263131 Email: guilhermessantos042@gmail.com

3 Mestrando em Administração com ênfase em Finanças pela Universidade Federal de Uberlândia. Brasil. Afiliação: Universidade Federal de Uberlândia. Lattes: http://lattes.cnpq.br/8358528670263131 Email: guilhermessantos042@gmail.com

4 Pós-Doutorado em Administração pela FEA, Universidade de São Paulo (2006). Brasil. Afiliação: Universidade Federal de Uberlândia. Lattes: http://lattes.cnpq.br/4700574397199469 Email: kribeiro@ufu.br 
Quando um gestor precisa tomar uma decisão, ele se depara com quatro principais dificuldades, segundo Roldan e Miyake (2004): complexidade, incerteza inerente a decisão, objetivos múltiplos interrelacionados e perspectivas diferentes, que podem levar a diferentes conclusões. Frente a isso, no processo de escolher o caminho mais adequado, os administradores veem a necessidade de utilizarem um fluxo constante e expressivo de informações e, para isso, utilizam as mais diversas ferramentas para analisar suas decisões (REZENDE, 2002).

A análise de decisão se distribui sobre dois extremos, o determinismo e a pura incerteza, em que o intervalo dessa interseção representa as questões voltadas ao risco. Como auxílio para a tomada de decisão, existem dois modelos de referência: modelos determinísticos e modelos probabilísticos. No primeiro, a decisão se baseia somente no resultado. Já o segundo considera, além do resultado, a quantidade de risco envolvida em cada decisão (DAMGHANI; TAGHAVIFARD; MOGHADDAM, 2009).

Como as empresas vivem em um cenário de contínua incerteza, analisar os riscos envolve o desenvolvimento de distribuições de probabilidade para a mensuração da eficácia (KAHRAMAN; RUAN; TOLGA, 2002). Segundo Martins (2001), o enfoque determinístico tradicional revela-se insuficiente quando nos deparamos com os problemas estratégicos vivenciados pelas empresas, pleno de incertezas e riscos. Uma alternativa para estimar os fluxos de caixa futuros é o valor presente líquido do Fluxo De Caixa Livre - FCL em condições de risco. Assim, a inserção de métodos aleatórios para os cálculos probabilísticos permite verificar de forma mais objetiva a ocorrência e a distribuição dos valores.

Nesse contexto, apresenta-se a questão da pesquisa: Quais as vantagens de se analisar uma empresa pelo método estocástico em detrimento aos métodos determinísticos convencionais? Para responder esee questionamento, o presente trabalho tem como objetivo fazer uma avaliação de uma empresa do setor siderúrgico por meio do método do fluxo de caixa descontado, sob a ótica da análise estocástica, a partir da simulação de cenários com auxílio do método de Monte Carlo. O setor foi escolhido por ser reconhecido pela sua importância no desenvolvimento econômico das nações, por fornecer insumos para infraestrutura, suprindo indústrias de construção, de bens de capital e de consumo, especialmente a automobilística (ANDRADE et al., 2001).

Com o objetivo de avaliar como os ativos financeiros e operacionais atuam na criação de valor em um cenário incerto, foi realizada uma avaliação do comportamento dos fluxos de caixa descontados da empresa Gerdau S.A. para um período de 5 anos, considerando a variação da taxa de crescimento do setor siderúrgico. Assim, sugere-se que, com a utilização de análises estocásticas, que levam em consideração o risco presente no processo decisório, evidencie uma análise que forneça aos gestores uma maior gama de informações que poderiam auxiliar na tomada de decisão mais acurada.

\section{Revisão da literatura}

Esta seção discute as teorias que envolvem a avaliação de empresas e os fatores envoltos nas abordagens determinísticas e probabilísticas, e como essas técnicas atuam nas decisões voltadas para a maximização do desempenho.

\subsection{Avaliação de empresas}

As informações contábeis, que registram as transações das empresas e correspondem a base de informações para os usuários, possuem limitações na mensuração e na evidenciação do valor patrimonial, devido a serem fundamentadas pelo custo histórico, fato que não demonstra o real valor econômico e de mercado de uma empresa (MÜLLER, 2004). Nesse sentido, a própria avaliação pelo valor contábil é pouco utilizada, sendo mais considerada em situações específicas, como casos de liquidação judicial ou empresas sem fluxo de caixa positivo, vista a limitação da apuração pelos custos históricos (DE JESUS; SEBASTIÃO; GIMENES, 2013).

Da Silva e Da Costa (2017) abordam que uma métrica de sinalização para capturar a qualidade da informação correspondente a participação no índice de sustentabilidade empresarial, que permite ganhos aos usuários em termos de redução de custos de transação. Contudo, Castro (2017) indica que não existe 
diferença significante no desempenho financeiro das empresas participantes e não participantes do índice de sustentabilidade empresarial.

O processo de avaliar uma empresa envolve uma série de decisões e também variáveis subjetivas. Para Müller e Teló (2003), tanto os usuários internos quanto os externos buscam uma avaliação de desempenho que se aproxime do valor justo de mercado. A maioria das pessoas muitas vezes fazem escolhas por hábito ou tradição, sem passar pelas etapas do processo de tomada de decisão sistematicamente. As decisões podem ser feitas sob pressão social, ou com restrições de tempo, que interfere na consideração das opções e nas consequências. Mesmo quando as pessoas têm tempo e informações, não conseguem interpretar e analisar as probabilidades de ocorrência, sendo mais propensas a confiar na experiência pessoal do que na informação sobre probabilidades. As preocupações fundamentais da tomada de decisão são a combinação de informações sobre probabilidades e informações sobre desejos e interesses (DAMGHANI; TAGHAVIFARD; MOGHADDAM, 2009).

Dentre os modelos de avaliação, os baseados no fluxo de caixa descontado correspondem ao que melhor revelam a efetiva capacidade de geração de riqueza de determinado empreendimento (DE JESUS; SEBASTIÃO; GIMENES, 2013; JUNIOR; CORREIA; GIMENES, 2015; MARTELANC et al., 2005). Na literatura, tem sido recomendada a utilização do método de desconto do $\mathrm{FCL}$, demonstrando eficiência esperada do empreendimento (MARTINS 2001).

\subsection{Fluxo de caixa livre}

Assaf Neto (2003) aborda que uma empresa tem que ser avaliada por sua riqueza econômica a valor presente, apreciada pelos benefícios esperados de caixas futuros e descontados por uma taxa de atratividade que corresponda ao custo de oportunidade das várias fontes de capital.

Prevalecendo como valor base para avaliação de empresas, na literatura e em pesquisas recentes (CUNHA et al., 2013; FERNÁNDEZ, 2007), o FCL é o excedente requerido de fluxo de caixa para financiar todos os projetos que tenham um valor presente líquido positivo, quando descontado por um relevante custo de capital. Em corroboração, Richardson (2006) define o FCL como o fluxo de caixa além do que é necessário para manter os ativos e ainda financiar novos investimentos esperados.

Brown (1996) distingue o FCL do fluxo de caixa tradicional, pois o primeiro é proveniente das operações correntes e está disponível para distribuição, não afetando os correntes níveis de crescimento da empresa. Assim, o excedente de caixa gerado efetivamente encontra-se disponível para a distribuição ou aumento de capital.

No quadro 1 está representado a forma contábil do FCL de acordo com Martins (2001).

Quadro 1 - Fluxo de caixa livre - FCL

(=) Receitas líquidas de vendas

(-) Custo das vendas

(-) Despesas Operacionais

$\Leftrightarrow$ Lucro antes dos juros e tributos sobre o lucro (EBIT)

(+) Ajuste de despesas operacionais que não provocam a saída de caixa

$\Leftrightarrow$ Lucro antes de juros, impostos sobre o lucro, depreciação, amortização e exaustão (EBITDA)

(-) Impostos de Renda/Contribuição Social

$\Leftrightarrow$ Geração de Caixa Operacional

(-) Investimentos

Permanentes

Circulantes

(=) Fluxo de Caixa Livre

Fonte: Martins (2001) 
O valor de um ativo é o fluxo de caixa que ele gera, descontado pelo custo de oportunidade representado pela soma da taxa livre de risco, com o adicional que representa o risco próprio desse ativo (MARTINS; MARTINS, 2015). Segundo Fernández (2007), a taxa apropriada a ser usada no desconto dos fluxos de caixa da empresa é o custo médio ponderado de capital (representado pela sigla WACC Weighted Average Cost of Capital), que corresponde à média ponderada da remuneração exigida pelas fontes de capital da empresa, acionistas e credores.

Conforme Müller e Teló (2003), o cálculo do valor da empresa com o uso dos modelos baseados no fluxo de caixa prevê, então, que o valor da estrutura de capital total, o valor de mercado, é igual ao valor presente do fluxo de caixa, considerado o WACC. A seguinte fórmula é sugerida por Assaf Neto (2003) para o cálculo do WACC líquido do imposto de renda:

$W A C C=(K e x W P L)+(K i x W P)$

em que:

$\mathrm{Ke}=$ custo de oportunidade do capital próprio;

$W P L=$ proporção do capital próprio $[P L / P+P L]$;

$\mathrm{Ki}=$ custo do capital de terceiros;

$\mathrm{WP}=$ proporção do capital (oneroso) de terceiros [P/P+PL];

$\mathrm{P}, \mathrm{PL}=$ respectivamente, passivo oneroso e patrimônio líquido (fundos próprios).

De acordo com Martins (2001), no valor presente do fluxo de caixa, em condições de risco, a taxa mínima de atratividade, que é a taxa de desconto, é considerada de modo determinístico. Damodaran (2007) demonstra o valor da empresa pelo fluxo de caixa descontado a partir da seguinte fórmula:

$$
\text { Valor de uma empresa }=\sum_{t=1}^{t=\infty} \frac{F C F F_{t}}{(1+W A C C)^{t}}
$$

em que:

$\mathrm{FCFF}_{\mathrm{t}}=$ Fluxo de caixa livre para a empresa no ano t;

WACC $=$ Média ponderada de custo de capital.

\subsection{Métodos estocásticos no fluxo de caixa em condições de risco}

O risco, na vida, é probabilidade de obtermos resultado em algo que não nos agradará. Por exemplo, ao dirigir um carro em alta velocidade, corremos o risco de sermos multados, ou de causarmos um acidente. No contexto de avaliação, a definição de risco se torna mais ampla, referindo-se à probabilidade de obtermos um retorno de investimento que seja diferente do previsto, pode ser para maior, chamado de upside risk, ou para menor, chamado de downside risk (DAMODARAN, 2007).

O processo de avaliação de um ativo, mesmo sendo efetuado a partir de modelos matemáticos, envolve aspectos subjetivos e, por consequência, é suscetível a incertezas e erros. Essas incertezas estão relacionadas, principalmente, à subjetividade das informações utilizadas no processo, pois o retorno de um ativo é calculado com base na expectativa de resultados futuros esperados para esse mesmo ativo. Nesse sentido, as informações de entrada são fundamentais e devem ser bem avaliadas para que o resultado da avaliação reflita o valor econômico justo da entidade avaliada (ENDLER, 2004).

Os métodos estocásticos correspondem aos processos em que, para cada valor das variáveis de entrada de um modelo, vai existir uma distribuição de probabilidades dos valores a serem assumidos pela variável dependente, a qual varia com o tempo, tornando as variáveis, em parte, aleatórias (ROGERS; SANTOS; LEMES, 2008). Uma modelagem probabilista para contemplar os aspectos da aleatoriedade da entrada das variáveis do risco no cálculo dos valores presentes do fluxo de caixa é o método de Monte Carlo, o qual, de acordo com Rubinstein e Kroese (2016), corresponde a uma modelagem estatística que se utiliza de uma sequência de números randômicos para gerar uma simulação. 
A simulação de Monte Carlo é, em essência, a geração de processos aleatórios por meio de um computador. Esses processos poderiam surgir naturalmente, como parte da modelagem de um sistema da vida real, como uma rede rodoviária complexa, o transporte de nêutrons ou a evolução do mercado de ações. Em muitos casos, no entanto, os objetos aleatórios nas técnicas de Monte Carlo são introduzidos artificialmente para resolver problemas puramente deterministas. Nesse caso, a simulação de Monte Carlo simplesmente envolve amostragem aleatória de certas distribuições de probabilidade. Tanto na configuração natural quando na artificial das técnicas de Monte Carlo, a ideia é repetir o experimento muitas vezes (ou usar uma corrida de simulação suficientemente longa) para obter muitas quantidades de interesse usando a Lei dos Grandes Números e outros métodos de inferência estatística (KROESE et al., 2014).

\subsection{Setor siderúrgico e a Gerdau S.A.}

O setor siderúrgico sempre foi reconhecido pela sua importância no desenvolvimento econômico das nações, por fornecer insumos para infraestrutura, suprindo indústrias de construção, de bens de capital e de bens de consumo, especialmente a automobilística (ANDRADE et al., 2001).

O Brasil tem importante participação no cenário internacional, sendo o único país fora da OCDE (Organização para Cooperação e Desenvolvimento Econômico) a fazer parte do seu Comitê do Aço. Além de possuir vantagens em termos de custo de mão-de-obra e custo de minério de ferro em função da abundância de jazidas existentes no país (RODRIGUES; CAMPOS, 2008).

O parque produtor siderúrgico brasileiro é composto por 29 usinas, administradas por 11 grupos empresariais, são eles: Aperam, ArcelorMittal Brasil, CSN, Gerdau, SINOBRAS, Thyssenkrupp CSA, Usiminas, VSB Tubos, Vallourec, Villares Metals e Votorantim. De acordo com os dados econômicos consolidados do setor, segundo levantamento realizado em 2015 pelo Instituto Aço Brasil, o Brasil possui o maior parque industrial de aço da América do Sul, sendo o maior produtor da América Latina e o sexto do mundo, e adiciona ao saldo comercial US\$ 3,5 bilhões e emprega mais de 110 mil colaboradores (INSTITUTO AÇO BRASIL, 2017).

Os riscos inerentes ao setor siderúrgico estão atrelados a sua característica cíclica, que ocasiona grande oscilação nos preços e altas demandas no mercado. Tal variabilidade é potencializada pela estrutura operacional alavancada do setor, com forte participação de custos fixos, gerando grande volatilidade nos retornos em projetos siderúrgicos, bem como nas ações de empresas do segmento. Essa volatilidade, em grande parte, pode ser explicada por três características da indústria: a fragmentação da estrutura do setor, pela inconstância observada na demanda dos seus principais mercados consumidores, e pela baixa flexibilidade no ajuste das quantidades ofertadas de significativa parcela dos produtores de aço (DE MAGALHÃES OZORIO, 2010).

O setor de metalurgia e siderurgia vêm apresentando oscilações referente a produção nos últimos anos. A produção mundial de aço bruto, em 2015, apresentou recuo de $3 \%$ em relação ao ano anterior e o Brasil teve redução de $1,8 \%$, colocando-se na $8^{a}$ posição do ranking mundial. O saldo comercial brasileiro do setor siderúrgico, em 2015, apresentou superávit de US\$ 3,5 bilhões. As exportações, em termos de volume, tiveram um crescimento de $40 \%$, enquanto o total em valor recuou $3 \%$, decorrente da deterioração dos preços do aço no mercado mundial. O consumo aparente de produtos siderúrgicos fechou o ano de 2015 com queda de $17 \%$ em relação a 2014, atingindo 21,2 milhões de toneladas. O Instituto Aço Brasil evidência que esses números são reflexo do cenário político-econômico nacional e da contínua perda de competitividade sistêmica que atinge a indústria brasileira do aço, além de outros fatores, como o excesso de capacidade de produção de aço no mundo (ANUÁRIO ESTATíSTICO DO SETOR METALÚRGICO, 2017).

De Magalhães Ozorio (2010) indica que a elasticidade da demanda do aço frente ao crescimento econômico possui dependência de setores extremamente cíclicos: construção civil, automobilístico e indústria de bens de capital. O mesmo autor aborda que a concorrência dentro do setor siderúrgico é bastante forte, fazendo com que os produtores de aço possam ser considerados tomadores de preço. 
A Gerdau tem a liderança no segmento de aços longos nas Américas e é uma das principais fornecedoras de aços longos especiais do mundo, possuindo operações em 12 países nas Américas e Ásia. Sendo a maior recicladora da América Latina e uma das maiores do mundo, transformando milhões de toneladas de sucata em aço (BRADESCO, 2017). A mesma apresenta características únicas, que a fizeram manter-se competitiva ao longo de sua trajetória, tais como senso de oportunidade, agilidade na adaptação das operações às oscilações de mercado, pioneirismo e inovação em diversas áreas. Essas competências foram fundamentais para garantir a sua sustentabilidade diante de um cenário de recessão econômica no Brasil, excesso de oferta global de aço, especialmente da China, e redução do consumo de aço em importantes mercados (GERDAU, 2017).

\section{Metodologia}

Para delimitar a amostra foram levantados os dados das demonstrações financeiras da empresa Gerdau S.A. e previsto o FCL de cinco períodos à frente. Os dados foram coletados entre o período de 03/2017 a 04/2017, e foram trabalhados os dados disponíveis entre o período de 01/2012 até 12/2016. A fonte de dados utilizada foi a base de dados Economática, sendo retiradas as empresas financeiras devido as suas peculiaridades contábeis.

Com a finalidade de alcançar o objetivo proposto neste estudo. foi aplicado o método de Monte Carlo para gerar a aleatoriedade dos dados em relação ao valor presente do FCL e verificar as probabilidades de ocorrências dos fluxos de caixa descontados nos próximos cinco períodos.

De acordo com o Estudo Prospectivo do Setor Siderúrgico (2008), o setor é um grande consumidor de recursos naturais e gerador de rejeitos indesejáveis, havendo, portanto, grandes oportunidades para inovações capazes de mitigar seus impactos ambientais. Conforme o mesmo documento, a sustentabilidade e competitividade se apresentam como questões que precisam ser consideradas pelas empresas, em que a primeira coloca a empresa num universo de comprometimento com as gerações futuras, via equilíbrio econômico, ambiental e ecológico, e sa segunda torna a empresa incapaz de frequentar os mercados e, por consequência, negaria a primeira.

A empresa em estudo foi escolhida pelo fato de se destacar por seus diferenciais competitivos, em que o seu mix de produtos envolve a produção de aços planos e minério de ferro. Além disso, é líder no segmento de aços longos nas Américas e uma das principais fornecedoras de aços especiais no mundo. Segundo a Worldsteel Association (2015), está entre as 20 líderes mundiais.

Em condições de incerteza, uma alternativa para a obtenção do retorno esperado e do risco de um projeto pode ser expresso a partir da utilização do método de Monte Carlo (BRUNI; FAMÁ; SIQUEIRA, 1998). Esse método probabilístico tem a vantagem de considerar o risco associado ao ato de projeção e mensurá-lo a partir do cálculo do desvio padrão das médias dos valores presentes líquidos gerados em cada evento (CORREIA NETO; MOURA; FORTE, 2002).

O método estocástico utilizado neste estudo está representado na figura 1.

Figura 1 - Modelo do método estocástico de análise

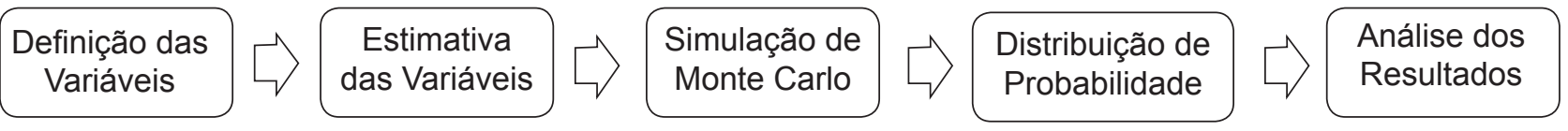

Fonte: Elaborada pelos autores

O processo de simulação pelo método de Monte Carlo efetua-se pela definição das variáveis nas quais ocorrerão os choques de aleatoriedade, alocando uma distribuição de probabilidade dada pelo histórico passado da variável. Depois do processo de identificação de cada variável, inicia-se o processo de geração de números aleatórios, conforme a faixa adotada para cada variável de entrada. Depois de gerados os valores para as variáveis de entrada (inputs), calcula-se automaticamente os valores das variáveis de saída (outputs) a partir das interações já firmadas entre elas, em que cada geração da série de números significa um cenário provável de ocorrência (ROGERS; SANTOS; LEMES, 2008). 


\section{Resultados}

As tabelas 1 e 2 apresentam os dados necessários para o cálculo do VPL dos últimos cinco anos da empresa e a projeção de crescimento do WACC para se calcular o VPL do fluxo de caixa livre. Dados determinísticos apurados na própria base de dados utilizada.

Tabela 1 - Cálculo do Fluxo de Caixa Livre (R\$ Milhares)

\begin{tabular}{|c|c|c|c|c|c|c|c|c|c|c|}
\hline & Data & Vendas & CDO & EBIT & IR & NOPAT & Depr & Capex & ICP & FCL \\
\hline \multirow{5}{*}{ 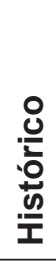 } & 2012 & 50.837 & 47.694 & 3.143 & 1.069 & 2.074 & 2.446 & 4.624 & 955 & -1.059 \\
\hline & 2013 & 50.377 & 46.896 & 3.481 & 1.184 & 2.297 & 2.565 & 3.417 & -1.164 & 2.609 \\
\hline & 2014 & 50.530 & 47.087 & 3.443 & 1.171 & 2.272 & 2.645 & 1.592 & 197 & 3.129 \\
\hline & 2015 & 46.768 & 50.218 & -3.451 & -1.173 & -2.277 & 2.799 & 2.599 & -1.508 & -570 \\
\hline & 2016 & 38.014 & 39.666 & -1.652 & -562 & -1.090 & 2.560 & 1.080 & -3.216 & 3.607 \\
\hline \multirow{5}{*}{$\begin{array}{l}\frac{0}{0} \\
\frac{\pi}{0} \\
\frac{0}{0} \\
\frac{0}{0}\end{array}$} & 2017 & 34.738 & 35.115 & -378 & -128 & -249 & 1.888 & 1.229 & 297 & 113 \\
\hline & 2018 & 31.743 & 32.210 & -467 & -159 & -308 & 1.847 & 1.202 & -649 & 986 \\
\hline & 2019 & 29.007 & 29.553 & -546 & -186 & -360 & 1.807 & 1.176 & -593 & 864 \\
\hline & 2020 & 26.507 & 27.122 & -616 & -209 & -406 & 1.768 & 1.151 & -542 & 753 \\
\hline & 2021 & 24.222 & 24.899 & -677 & -230 & -447 & 1.730 & 1.126 & -495 & 653 \\
\hline
\end{tabular}

Nota: Variáveis: Vendas = Vendas do período; $\mathrm{CDO}=$ Custos e Despesas Operacionais; EBIT = Lucros antes de juros e impostos; IR = Imposto de Renda; NOPAT = Lucro Operacional Líquido após os impostos; Depr = Depreciação; CAPEX = Despesas de Capital; ICP = Investimentos em Capital de Giro; FCL = Fluxo de Caixa Livre.

Fonte: Dados da pesquisa - 2017

Os valores referentes ao crescimento das vendas e EBITDA foram, respectivamente, de -8,6 e 4,3\%. A projeção do CAPEX e depreciação foi, respectivamente, de 6,2 e $65,1 \%$, sendo essas informações anualizadas. A alíquota de imposto de renda deste estudo corresponde a $34 \%$ ao ano, e no risco país foi considerado o valor do último ano da análise.

Tabela 2 - Projeção do WACC e do VPL do Fluxo de Caixa

\begin{tabular}{|c|c|c|c|c|c|c|c|c|c|}
\hline & Data & $\begin{array}{c}\text { Risk free } \\
\text { real \% } \\
\text { (USA) }\end{array}$ & $\begin{array}{c}\text { Prêmio } \\
\text { mercado \% } \\
\text { (USA) }\end{array}$ & $\begin{array}{c}\text { Beta } \\
\text { alavancado }\end{array}$ & $\begin{array}{l}\text { Risco } \\
\text { país \% }\end{array}$ & $\begin{array}{c}\text { Ke real } \\
\%\end{array}$ & $\begin{array}{c}\text { Kd real } \\
\%\end{array}$ & $\begin{array}{c}\mathrm{D} /(\mathrm{D}+\mathrm{E}) \\
\%\end{array}$ & $\begin{array}{l}\text { WACC } \\
\text { real \% }\end{array}$ \\
\hline \multirow{5}{*}{$\begin{array}{l}\frac{0}{0} \\
\frac{\pi}{0} \\
\frac{0}{2} \\
\frac{0}{2}\end{array}$} & 2017 & 0.44 & 6.0 & 1.9 & 3.8 & 15.5 & 2.6 & 62.7 & 7.4 \\
\hline & 2018 & 0.44 & 6.0 & 1.9 & 3.8 & 15.5 & 2.6 & 62.7 & 7.4 \\
\hline & 2019 & 0.44 & 6.0 & 1.9 & 3.8 & 15.5 & 2.6 & 62.7 & 7.4 \\
\hline & 2020 & 0.44 & 6.0 & 1.9 & 3.8 & 15.5 & 2.6 & 62.7 & 7.4 \\
\hline & 2021 & 0.44 & 6.0 & 1.9 & 3.8 & 15.5 & 2.6 & 62.7 & 7.4 \\
\hline \multicolumn{4}{|c|}{ Valor Presente do Fluxo de Caixa } & 2.682 & & & & & \\
\hline
\end{tabular}

Nota: Variáveis: Risk free real = Taxa do ativo livre de risco; Prêmio mercado = Prêmio pelo ativo livre de risco; Beta alavancado = Indicador de sensibilidade do ativo; Risco país = Risco Brasil; Ke real = Custo capital próprio; Kd real =Custo capital de terceiros; WACC = Custo Médio Ponderado de Capital.

Fonte: Dados da pesquisa - 2017

A variável referente ao crescimento projetado foi tratada a partir dos dados coletados, utilizando-se como referência o crescimento projetado da empresa e como variação a diferença entre o crescimento do setor e o crescimento projetado. A partir desses dados, foram realizadas cem mil simulações pelo método de Monte Carlo de cenários utilizando o software Crystal Ball@ para verificar o comportamento do VPL do fluxo de caixa, sendo o resumo da simulação apresentado na tabela 3. 
Tabela 3 - Resumo da Simulação de Monte Carlo (R\$ Milhares)

\begin{tabular}{lccc}
\hline Estatísticas & \multicolumn{2}{c}{ Percentil } \\
\hline Simulação & Monte Carlo & $\mathbf{1 0 \%}$ & -2.206 \\
Número de Iterações & 100.000 & $\mathbf{2 0} \%$ & -516 \\
Mínimo & -13.005 & $\mathbf{3 0 \%}$ & 691 \\
Máximo & 18.776 & $\mathbf{4 0 \%}$ & 1.724 \\
Média & 2.698 & $\mathbf{5 0 \%}$ & 2.702 \\
Mediana & 2.702 & $\mathbf{6 0 \%}$ & 3.665 \\
Desvio Padrão & 3.815 & $\mathbf{7 0 \%}$ & 4.701 \\
Variância & 14.554 .003 & $\mathbf{8 0} \%$ & 5.920 \\
Coeficiente de Variação & $1,41 \%$ & $\mathbf{9 0 \%}$ & 7.603 \\
\hline
\end{tabular}

Fonte: Dados da pesquisa -2017

Apartir desses resultados foi possível definir uma distribuição normal que demonstrou o comportamento do VPL dos fluxos de caixa, conforme demonstrado no gráfico 1. A análise dos dados a partir da simulação de Monte Carlo demonstra o comportamento do VPL dos fluxos de caixa médio de cem mil simulações e foi possível inferir com $90 \%$ de confiabilidade que o valor se encontra no intervalo de $\mathrm{R} \$-3.580$ e $\mathrm{R} \$$ 8.976.

Gráfico 1 - Distribuição Normal Gerdau S.A. (R\$Milhares)

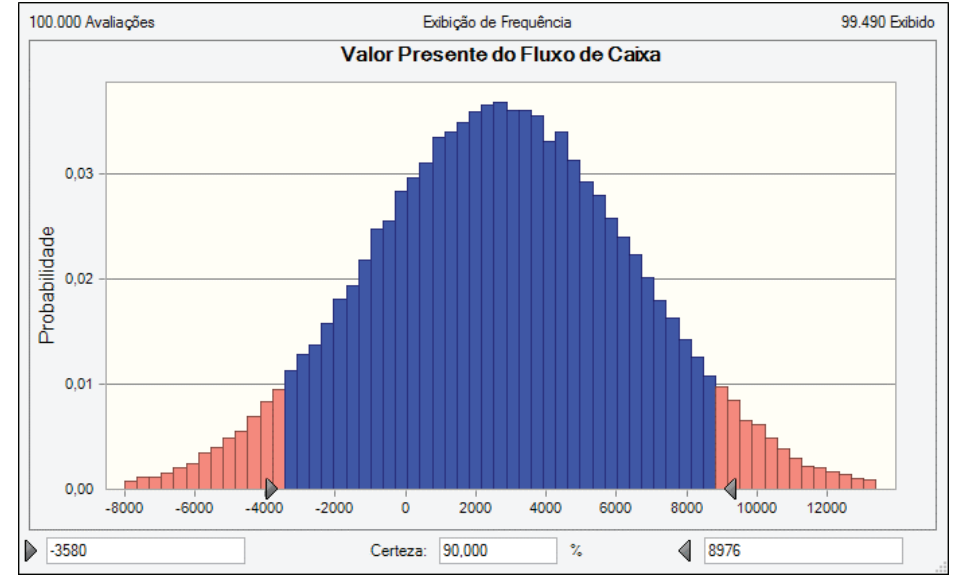

Fonte: Dados da pesquisa-2017

Ao analisar os dois métodos de avaliação, determinístico e estocástico, é possível inferir, pelo método estocástico utilizado, tendo como base o valor determinístico do VPL de R 2.682 (apresentado na tabela 2), que 50,21\% dos valores vão estar localizados abaixo desse patamar fixo, conforme gráfico 2 . Analisando esse valor com base nos resultados do modelo estocástico, infere-se que a empresa apresenta $50,21 \%$ de chances de não obter crescimento acima da projeção determinística do setor de siderurgia e, com isso, incorrer em perda de competitividade, por não conseguir acompanhar o crescimento do setor. 
Gráfico 2 -Valor Presente do Fluxo de Caixa - Gerdau S.A. (R\$ Milhares)

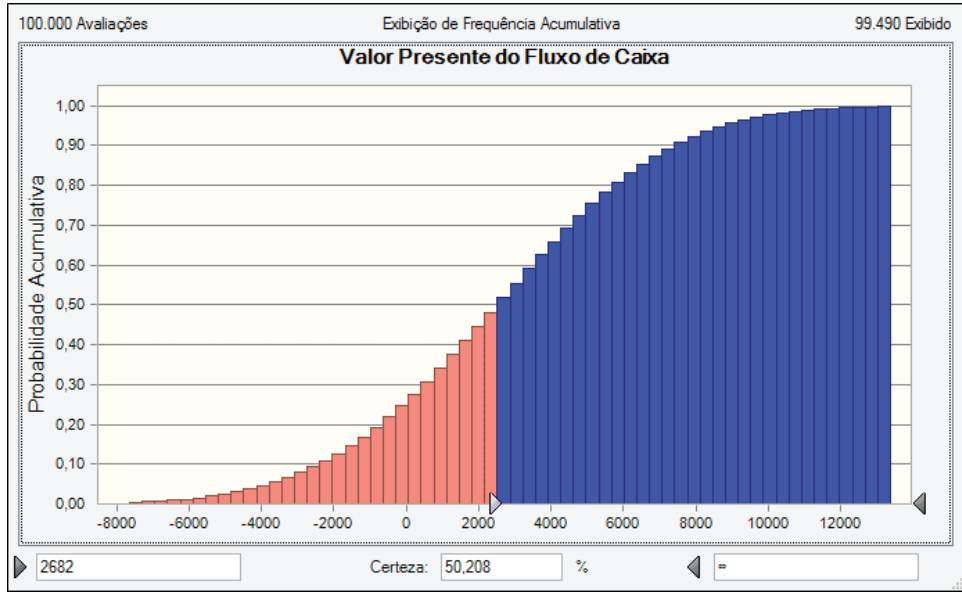

Fonte: Dados da pesquisa - 2017

Devido a apresentar oscilações referentes ao crescimento em seus dados históricos, constata-se a probabilidade de ocorrência do VPL do fluxo de caixa negativo para os próximos cinco anos, o qual, neste estudo, foi de $24 \%$, sendo apresentado no gráfico 3 , demonstrando que o cenário projetado para o setor e para a empresa se encontra envolto em incertezas.

Gráfico 3 - Probabilidade de Valor Presente do Fluxo de Caixa Negativo - Gerdau S.A. (R\$ Milhares)

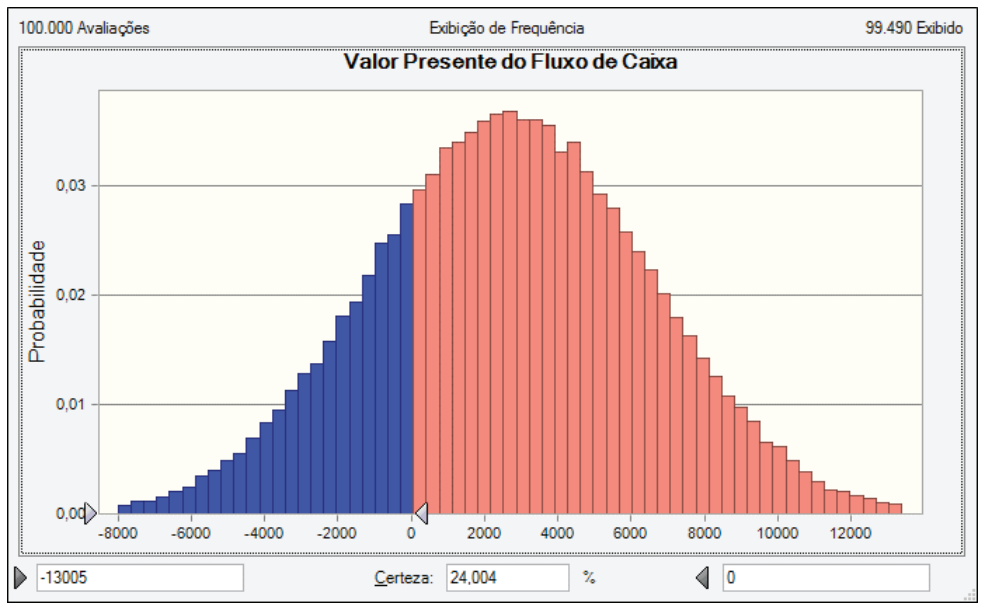

Fonte: Dados da pesquisa - 2017

Esse resultado demonstra que os métodos determinísticos negligenciam as variações entre períodos, as quais representam os fatores de risco inerentes nas empresas e podem, assim, superestimar ou subestimar as informações projetadas de acordo com o viés de crescimento da empresa referente ao período analisado, tendo como base dados históricos.

Sobre o componente estocástico da análise, Andersen \& Bettis (2015) afirmam que pode resultar da acumulação de erros inevitáveis e resultados aleatórios decorrentes de processos de aprendizagem experiencial como parte da adaptação organizacional. Reforçando: por maior que seja o cuidado do gestor em tentar prever todas as variáveis a que está sujeito o mercado, sempre existe um componente ou situação não possível de determinar e controlar. Os mesmos autores ainda ressaltam que modelar matematicamente ou estatisticamente os sistemas e processos racionais, mas interdependentes - portanto, altamente nãolineares dentro das organizações -, podem produzir resultados aparentemente aleatórios que são uma avenida potencial e importante para futuras pesquisas.

Os resultados corroboram com os estudos de Bruni, Famá e Siqueira (1998), Correia Neto, Moura e Forte (2002), Rogers, Santos e Lemes (2008) e De Magalhães Ozorio (2010) ao avaliar as empresas pela ótica probabilística para evidenciar os aspectos do risco. Sendo assim, quando a empresa avalia, por meio do VPL dos FCL projetados, ela busca medir se as suas estratégias serão eficientes no futuro e como 
ocorrerá esse comportamento de preferência. Já ao incluir a variável risco em suas análises, elas ganham um suporte importante em suas tomadas de decisões.

Os resultados também vão ao encontro de Kroese et al. (2014), em que a medida que os produtos financeiros continuam a crescer em complexidade, as técnicas de Monte Carlo tornam-se ferramentas cada vez mais importantes para analisá-los. A simulação de Monte Carlo não é usada apenas para avaliar instrumentos financeiros, também desempenha um papel crítico na análise de risco. Essas técnicas são particularmente eficazes na resolução de problemas envolvendo várias fontes de incerteza. Uma grande força das técnicas de Monte Carlo para análise de risco é que elas podem ser facilmente usadas para executar a análise de cenários - isto é, podem ser usadas para calcular os resultados de risco sob uma série de diferentes pressupostos do modelo.

Além disso, em relação ao gerenciamento de riscos, Eckert e Gatzert (2015) indicam que se deve colocar especial ênfase nesses eventos e implementar medidas efetivas para reduzir sua probabilidade e impacto. Além disso, ainda sobre os resultados desses autores, quando se considera o risco no processo de avaliação para a tomada de decisão, permite-se que se realize o seu gerenciamento, dando a devida ênfase nos aspectos relacionados ao risco e à implementação de medidas efetivas para reduzir sua probabilidade e impacto.

\section{Conclusão}

Com fins de evidenciar a eficiência da utilização de métodos estocásticos em detrimento de modelos determinísticos para estimar o comportamento do VPL do FCL de empresas, este estudo aplicou o método de Monte Carlos sobre os dados da empresa Gerdau S.A. visando prever o comportamento dos seus números para os próximos cinco anos.

Com a utilização do método de Monte Carlo foi possível obter informações mais abrangentes referentes a incertezas que envolvem o processo decisório em cenários empresariais altamente competitivos. Tal procedimento permitiu avaliar os dados de acordo com as suas probabilidades de ocorrência e de forma aleatória, processo que se apresenta com maior utilidade frente aos métodos determinísticos.

Avaliar empresas se apresenta como uma função gerencial capaz de verificar como a tomada de decisão atua na criação de valor e na competitividade das empresas. Para isso, os gestores devem considerar cenários em que a presença de riscos é realidade. Para isso, a utilização de modelos probabilísticos pode gerar informações mais acuradas.

Os resultados deste estudo demonstram que, a partir da aplicação da simulação de Monte Carlo, foi possível atribuir aspectos estatísticos para evidenciar os fatores correspondentes aos riscos do setor siderúrgico. Após os choques de simulação de cenários aleatórios, foi possível inferir que os métodos estocásticos permitem uma avaliação mais acurada da empresa que os procedimentos determinísticos, evidenciando que, à medida que os produtos financeiros continuam a crescer em complexidade, as técnicas de Monte Carlo tornam-se ferramentas cada vez mais importantes para analisá-los

Entretanto, faz-se necessário reconhecer as limitações das simulações realizadas. Apesar das análises estocásticas se utilizarem de procedimentos aleatórios e probabilísticos, não é possível determinar todas as variáveis que podem impactar nos fluxos de caixa projetados, além dos dados projetados serem oriundos de fontes determinísticas. Sugere-se para futuras pesquisas a utilização de métodos que permitam verificar a interação entre as variáveis e os riscos sistêmicos para um melhor ajustamento do modelo.

\section{Referências}

ANDERSEN, Torben J.; BETTIS, Richard A. Exploring longitudinal risk return relationships. Strategic Management Journal, [S.I], v. 36, n. 8, p. 1135-1145, 2015.

ANUÁRIO SETOR METALÚRGICO ESTATÍSTICO, (Brasil). Secretaria de Minas e Metalurgia-

SMM. Ministério de Minas e Energia-MME. 2016. Disponível em: <http://www.mme.gov.br/ documents/1138775/1732813/ANU\%C3\%81RIO+METAL\%C3\%9ARGICO+2016_vers\%C3\%A30+3. pdf/9595d304-7072-4313-a190-b5b97a10e7a5>. Acesso em: 24 set. 2017. 
ANDRADE, M. L. A. D. et al. Impactos da privatização no setor siderúrgico. BNDES Estudos Setoriais, n. 2, 2001.

ANDREEVA, T.; KIANTO, A. Does knowledge management really matter? Linking knowledge management practices, competitiveness and economic performance. Journal of Knowledge Management, [S.I], v. 16, n. 4, p. 617-636, 2012.

ASSAF NETO, A. Finanças Corporativas e Valor. São Paulo: Atlas, 2003.

BRADESCO. (Brasil). Laudo de avaliação no contexto da oferta pública de aquisição de ações por aumento de participação na Gerdau S.A. 2017. Disponível em: <http://ri.gerdau.com/ptb/7556/ Anexo\%203\%20-\%20Laudo\%20de\%20Avaliao.pdf>. Acesso em: 27 abr. 2017.

BROWN, G. T. “Free Cash Flow” Appraisal... A Better Way?. Appraisal Journal, [S.I], v. 64, p. 171-182, 1996.

BRUNI, A. L.; FAMÁ, R.; SIQUEIRA, J. de O. Análise do risco na avaliação de projetos de investimento: uma aplicação do método de Monte Carlo. Caderno de pesquisas em Administração, [S.I], v. 1, n. 6, p. 1, 1998.

CASTRO, L. A. Análise de diferenças de desempenho entre empresas participantes e não participantes do Índice de Sustentabilidade Empresarial da BM\&FBOVESPA. Revista Ciências.Fortaleza, v. 23, n. 1, p. 128-155, já./abr.2017.

CORREIA NETO, J. F.; MOURA, H. J. D..; FORTE, S. H. A. C. Modelo prático de previsão de fluxo de caixa operacional para empresas comerciais considerando os efeitos do risco, através do Método de Monte Carlo. Revista Eletrônica de Administração, Porto Alegre/RS, Edições 27, v. 8, n. 3, p.1-23, maio./jun.2002.

CUNHA, M. F. et al. Custo de Capital Médio Ponderado na Avaliação de Empresas no Brasil: Uma investigação da aderência acadêmica e a prática de mercado. Revista Ambiente Contabil, Natal-RN, v. 5, n. 2, p. 20-36, jul./dez, 2013.

DA SILVA, G. R.; DA COSTA, F. M. Qualidade da informação contábil e sustentabilidade nas companhias brasileiras listadas na BM\&FBovespa. Revista Ciências Administrativas, Fortaleza, v. 23, n. 1, p. 103127, jan./abr.2017.

DAMGHANI, K. K.; TAGHAVIFARD, M. T.; MOGHADDAM, R. T. Decision making under uncertain and risky situations. In: Enterprise Risk Management Symposium Monograph Society of ActuariesSchaumburg, Chicago, Illinois.2009. p.1-22.

DAMODARAN, A. Avaliação de Empresas. 2. ed. São Paulo: Pearson, 2007.

DE JESUS, M.; SEBASTIÃO, G. C.; GIMENES, R. M. T. Metodologias de avaliação de empresas. Revista de Ciências Empresariais da UNIPAR, Umuarama, v. 13, n. 2, p. 279-294, jul./dez. 2013.

DE MAGALHÃES OZORIO, L. Opções reais na Siderurgia: o caso brasileiro. 208f. 2010. Tese (doutorado) - Pontifícia Universidade Católica do Rio de Janeiro, Departamento de Engenharia Industrial, Rio de Janeiro, 2010

ECKERT, C.; GATZERT, N. Modeling operational risk incorporating reputation risk: An integrated analysis for financial firms. Insurance: Mathematics and Economics, v. 72, n. C, p. 122-137, 2017.

ENDLER, L. Avaliação de empresas pelo método de fluxo de caixa descontado e os desvios causados pela utilização de taxas de desconto inadequadas. ConTexto, Porto Alegre, v. 4, n. 6, $1^{\circ}$ semestre, 2004.

ESTUDO PROSPECTIVO DO SETOR SIDERÚRGICO (Brasil). Panorama do Setor Siderúrgico. 2008. Disponível em: <http://www.abmbrasil.com.br/epss/arquivos/documentos/2011_4_18_16_42_43_32042. pdf>. Acesso em: 27 abr. 2017.

FERNÁNDEZ, P. et al. Company valuation methods. The most common errors in valuations. IESE-CIIF, WP n.449, p. 1-30, Feb., 2007. 
GERDAU S.A. (Brasil). Relato Integrado. 2016. Disponível em: <http://gerdau.infoinvest.com.br/ ptb/7547/2016\%20-\%20relato-integrado-gerdau.pdf>. Acesso em: 27 abr. 2017.

INSTITUTO AÇO BRASIL (Brasil). Dados consolidados do setor siderúrgico. 2015. Disponível em: <http://www.acobrasil.org.br/site2015/dados.asp>. Acesso em: 26 abr. 2017.

JUNIOR, J. L. B.; CORREIA, E. F.; GIMENES, R. M. T. Avaliação de Empresas pelo Método do Fluxo de Caixa Descontado: o caso de uma indústria de ração animal e soluções em homeopatia. Contabilidade Vista \& Revista, Universidade Federal de Minas Gerais, Belo Horizonte , v. 26, n. 2, p. 90-113, maio/ ago. 2015.

KAHRAMAN, C.; RUAN, D.; TOLGA, E. Capital budgeting techniques using discounted fuzzy versus probabilistic cash flows. Information Sciences, [S.I], v. 142, n. 1-4, p. 57-76, May 2002.

KROESE, D. P. et al. Why the Monte Carlo method is so important today. Wiley Interdisciplinary Reviews: Computational Statistics, [S.I], v. 6, n. 6, p. 386-392, Nov./Dec. 2014.

MARTELANC, R. et al. Utilização de metodologias de avaliação de empresas: resultados de uma pesquisa no Brasil. In:SEMINÁRIO EM ADMINISTRAÇÃO, FEA/USP-SEMEAD, 8ª, 2005.Anais... São Paulo: FEA/USP, p. 1-12, 2005.

MARTINS, E. (org.) Avaliação de Empresas:da mensuração contábil à econômica. São Paulo: Atlas, 2001.

MARTINS, E.; MARTINS, V. A. Contabilidade e finanças: a temerária utilização do WACC. Revista Universo Contábil, Blumenau, v. 11, n. 1, p. 25-46, jan./mar., 2015

MÜLLER, A. N. Análise do valor das empresas: os limites dos modelos de avaliação. Revista FAE, Curitiba, v.7, n.2, p.73-82, jul./dez. 2004.

MÜLLER, A. N.; TELÓ, A. R. Modelos de avaliação de empresas. Revista da FAE, Curitiba v. 6, n. 2, p.97-112, maio/dez. 2003.

REZENDE, Y. Informação para negócios: os novos agentes do conhecimento e a gestão do capital intelectual. Ci. Inf., Brasília, Brasília, v. 31, n. 1, p. 75-83, jan./abr. 2002.

RICHARDSON, S. Over-investment of free cash flow. Review of accounting studies, [S.I], v. 11, n. 2-3, p. 159-189, Sep. 2006.

RODRIGUES, R. V.; CAMPOS, A. C. Desempenho e Competitividade do Setor Siderúrgico Brasileiro na década de 90. Revista de Economia, UEG, Anápolis-GO, v. 4, n. 01, p. 56-74, jan./jun. 2008.

ROGERS, P.; SANTOS, E. J.; LEMES, S. Precificação em empresas comerciais: um estudo de caso aplicando o custeio variável através do método de monte carlo. Revista da FAE, Curitiba, v.11, n.1, p.55-67, jan./jun. 2008.

ROLDAN, F.; MIYAKE, D. I. Mudanças de forecast na indústria automobilística: iniciativas para a estruturação dos processos de tomada de decisão e processamento da informação. Gestão \& Produção, [S.I], v. 11, n. 3, p. 413-427, set./dez. 2004.

RUBINSTEIN, R.; KROESE, D. Simulation and the monte carlo method. 2nd. ed. Canada: John Wiley \& Sons, 2016.

WORLDSTEEL ASSOCIATION. Top steel-producing companies. 2015. Disponível em: <https://www. worldsteel.org/steel-by-topic/statistics/top-producers.html>. Acesso em: 24 abr. 2017.

Submetido em: 09/08/2017

Aprovado em: 21/10/2017 